\title{
El programa Aprende en casa, ¿una estrategia que excluye a los usuarios de lenguas minoritarias?
}

\author{
MIROSLAVA CRUZ-ALDRETE* \\ Universidad Autónoma del Estado de Morelos (UAEM) - México \\ Recibido el 11-06-21; evaluado el 11-07-21; \\ aceptado el 10-08-21
}

\section{Resumen}

En este trabajo discuto la compleja situación a la que se enfrentaron los grupos lingüísticos minoritarios, en particular los sordos usuarios de la lengua de seńas mexicana (LSM), cuya enseńanza bilingüe se vio amenazada ante la crisis educativa ocasionada por la pandemia causada por el nuevo coronavirus. La respuesta del estado mexicano para no interrumpir la educación de los niños y jóvenes hizo patente la discriminación hacia los usuarios de lenguas diferentes al español, al ser esta la lengua vehicular para la programación de Aprende en casa, y no considerar el contexto y los idiomas de las comunidades indígenas y sordas, para su enseńanza-aprendizaje.

Palabras clave: lengua de señas, lenguas minoritarias, educación bilingüe.

\section{The Aprende en casa program, a strategy that excludes minority language users?}

\section{Abstract}

In this paper I discuss the complex situation faced by minority linguistic groups, in particular deaf users of the Mexican Sign Language (LSM), whose bilingual education was threatened by the educational crisis caused by the pandemic. The response of the Mexican state not to interrupt the education of children and young people in this country, made clear the discrimination against users of languages distinct of Spanish, as this is the vehicular language for the programming "Aprende en casa", and doesn't considerer the context and languages if the indigenous and deaf communities for their teaching.

Keywords: sign language, minority languages, bilingual education.

\footnotetext{
* Doctora en Lingüística por El Colegio de México (2008) con la tesis Gramática de la Lengua de Señas Mexicana. Profesora-investigadora del Centro Interdisciplinario de Investigación en Humanidades. Miembro del Sistema Nacional de Investigadores nivel II. Líneas de investigación: Estudios del Discurso (CA) y el estudio de la gramática de la lengua de señas mexicana. https://orcid.org/0000-0001-8110-4300. Correo electrónico: miroslava.cruza@uaem.edu.mx
} 


\section{O programa Aprenda em Casa, uma estratégia que exclui usuários de línguas minoritárias?}

\section{Resumo}

Neste artido discuto a complexa situação enfrentada por grupos lingüísticos minoritários, em particular surdos usuários da Língua de Sinais Mexicana (LSM), cuja educação bilíngue foi ameaçada pela crise educacional causada pela pandemia. A resposta do Estado mexicano em continuar com educação de crianças e jovens neste país, deixou clara a discriminação contra usários de outras línguas que não o espanhol, por ser esta a língua vehicular para a programação do Aprenda e não considera o contexto e línguas de comunidades índigenas e surdas, para seu ensino.

Palavras-chave: línguas de sinais, línguas minoritárias, educação bilíngue.

\section{INTRODUCCIÓN}

La crisis sanitaria provocada por el nuevo coronavirus enfrentó al mundo a circunstancias extraordinarias para continuar con la dinámica de la vida cotidiana a la que estábamos acostumbrados, sabiendo que esta ya no volvería a ser la misma. Las estrategias como el confinamiento y la sana distancia impactaron en muchos ámbitos, entre ellos, y de manera importante a «la educación» desde el nivel inicial, educación básica y superior.

Es quizá este ámbito, el educativo, el cual no solo puso en evidencia la brecha que existe entre las naciones con un mayor índice de desarrollo y aquellos que se han denominado países en vías de desarrollo, sino, además, puso de manifiesto las condiciones de marginación que por siglos han tenido, en nuestro caso particular, en un país como México, las comunidades indígenas y las comunidades sordas.

Resulta lamentable que persista la deuda histórica que se tiene con ambos grupos en cuanto al desarrollo de una política lingüística y educativa cuyo fin sea el fortalecimiento de su propia lengua. Hoy en día podemos observar que la puesta en marcha de un modelo educativo bilingüe para las comunidades lingüísticas minoritarias no responde la más de las veces a un respeto por el desarrollo de ambas lenguas (dominante y originaria) y ambas culturas. Por el contrario, la lengua materna del alumnado, indígena o sordo, no se ha valorado como el vehículo del pensamiento, de la construcción del conocimiento entre pares, y como el rasgo que da identidad a los miembros de la comunidad. Así, la primera lengua de los estudiantes suele ser utilizada como una herramienta para lograr el aprendizaje del español (oral y escrito), vestido este último como la lengua del conocimiento. 
La propuesta de la educación bilingüe para las poblaciones indígenas no es algo reciente, a diferencia de las comunidades sordas. Han recorrido caminos diferentes, pero, desafortunadamente, comparten la misma problemática. De ahí que resulte oportuno retomar la crítica que se ha hecho sobre la propuesta de la educación bilingüe en nuestro país, como bien lo señala Rebeca Barriga (2018) en su extraordinario libro De Babel a Pentecostés. Politicas lingüisticas y lenguas indígenas, entre historias, discursos, paradojas y testimonios. Los retos pueden ser los mismos en términos de conservar la lengua materna, enriquecerla, y aprender el espańol como una segunda lengua, sin embargo, en el caso de los sordos señantes, nos adentramos a una situación de mayor complejidad, por la diferencia de la modalidad de las lenguas que entran en contacto.

La obra de Barriga Villanueva toma realce para entender el estado actual de la educación bilingüe. Nos presenta de manera sucinta diversos acontecimientos sociohistóricos que definieron la construcción del México que hoy conocemos. Y, nos conduce a revisar cómo desde el siglo XVI hasta la actualidad, se ha tejido una compleja urdimbre en torno a la diversidad lingüística y a la enseñanza del español a los indígenas, en donde, el uso de la lengua materna ha sido empleada, principalmente, para aprender el espańol como la lengua dominante en detrimento de las minorías lingüísticas. Esto, sin lugar a duda, ha propiciado una tensión constante entre el español y las lenguas indígenas, así como la permanente oscilación de la política lingüística que desde hace siglos transita entre dos polos: «castellanización o lenguas indígenas, lenguas indígenas o castellanización» (Barriga, 2018, p. 23).

En este recorrido presenciamos la génesis de una política de estado cuyo ideal de nación se emparejaba con el principio de tener una sola lengua; y de igual manera la apuesta al progreso ligado a la desaparición de las culturas originarias. Para esta investigadora, si bien la última década del siglo $\mathrm{XX}$ trajo consigo nuevos aires para el reconocimiento de los derechos lingüísticos de las comunidades minoritarias, que condujo al desarrollo de una política intercultural, con el fin de dar una mejor atención a las demandas de las comunidades indígenas, entre ellas, la conservación de sus lenguas. Esto a su vez, trajo consigo una nueva propuesta educativa bilingüe (Quishpe Lema, 2001). Sin embargo, en los hechos, Barriga observa que este nuevo paradigma no está exento de contradicciones e incongruencia, de tal modo que sus resultados no han sido los esperados, como se refleja en los estudios reportados por el extinto Instituto Nacional de Evaluación Educativa (INEE). Por ejemplo, en 2019, el INEE al referirse a los logros de la educación para el alumnado indígena, encuentra que presentan los índices de aprendizaje más bajos del 
sistema educativo nacional, el porcentaje más elevado de reprobación y, una prematura deserción escolar.

Aun cuando son varios los factores a los cuales se podría atribuir estos resultados, desde la pobreza y la marginación en la que viven estas comunidades, así como la falta de recursos humanos, formación de profesores bilingües, o la carencia de materiales educativos adecuados, habría que considerar también el papel que juega la lengua en la enseñanza. La situación de diglosia en la que se encuentran los alumnos, la lengua de la escuela y la del hogar, el idioma utilizado en los espacios de la vida pública. Sin duda, es un factor relevante las actitudes lingüísticas de los miembros de las comunidades indígenas, hacia el fortalecimiento de su lengua materna, y hacia al aprendizaje del espańol como una segunda lengua. Así como el vínculo que se establece entre los contenidos curriculares que se ofertan en el aula y el saber de la comunidad. Por ende, el gran reto de la educación bilingüe intercultural es propiciar un espacio en donde se reconozca el conocimiento ancestral, la historia, las tradiciones, la cultura de los pueblos originarios, y la diversidad lingüística. Y, no menos importante, entender cuál ha sido la resistencia de estas comunidades ante al avasallante empuje del español, y de la cultura dominante.

Esta situación alcanza a los hablantes de lenguas indígenas y, a los sordos usuarios de una lengua de señas. Pareciera que no es opción para estos hablantes/señantes el monolingüismo, dada la compleja realidad en la que se desenvuelven y que demanda el aprendizaje del español con el fin de poder ejercer su derecho a la información, a la salud, a la justicia, al trabajo, a la educación.

La instrumentación de la enseñanza para estas comunidades lingüísticas minoritarias, entre ellas la conformada por los sordos usuarios de la lengua de señas mexicana (LSM), presenta una problemática educativa (cuyo abordaje rebasa los límites de este trabajo) que se vio agravada ante el cierre de los centros educativos como parte de las medidas sanitarias para evitar la transmisión de la COVID-19. Sirva el contexto enunciado para centrar la discusión sobre una de las medidas tomadas por la Secretaría de Educación Pública (SEP), para afrontar la crisis educativa ocasionada por la pandemia causada por la COVID-19, la cual nos ha mantenido por más de un año (a partir de marzo de 2020 a la fecha) alejados de las aulas. Esta acción se basó en el uso de la televisión educativa con la puesta en marcha del programa Aprende en casa.

La atención a los alumnos desde el nivel básico (preescolar, primaria y secundaria) se centró de manera importante en el seguimiento por parte de los alumnos y padres de familia a la barra de programas propios de cada nivel escolar, ofertados por el programa Aprende en casa. Aunado al acompaña- 
miento de los profesores, quienes dieron una pronta respuesta para organizarse con sus alumnos y poder atestiguar sus aprendizajes. Se trató de una estrategia qué si bien pudo favorecer a miles de estudiantes, por el contrario, hubo a quienes esta propuesta no les permitió continuar su formación.

No todos pudieron seguir «las clases» que se ofrecían en los varios canales por televisión abierta. Al respecto, el sector de la población que no se vio beneficiado por esta iniciativa fue aquella conformada por alumnos provenientes de comunidades rurales, indígenas, y sordas. Por un lado, debido a los problemas técnicos de acceso, es decir, la falta de una infraestructura adecuada para acceder a la señal de televisión o la red de internet, incluso, por carecer de energía eléctrica, o por no contar con los dispositivos necesarios. Y por el otro, porque no se tuvo en cuenta la condición lingüística de un sector de la población.

Como veremos más adelante, se puso en evidencia el desconocimiento de las autoridades, por omisión o comisión, sobre los estudiantes que tienen una lengua minoritaria, ya sea autóctona o de señas, que requerían no solo el cambio de modalidad presencial a una vía remota o virtual. Sino demandaba la creación de programas orientados a su condición lingüística, desde la perspectiva de la educación bilingüe.

Nos parece importante discutir, cómo este tipo de medidas educativas de manera soterrada favorecen la hegemonía del idioma español, como fue el caso la propuesta Aprende en casa, ya que desde una iniciativa gubernamental colocó a este idioma como la lengua de conocimiento, se le identificó como el idioma de la «escuela». Es indudable el gran reto que tiene la SEP para atender la demanda educativa en un país con una gran diversidad de lenguas y culturas, ante la falta de recursos humanos, estructurales, y económicos, para garantizar una educación bilingüe. Sin embargo, esto no debiera ser una excusa para no discutir el hecho que los alumnos que cursaban sus estudios desde el enfoque de un modelo bilingüe no tuvieron más opción que tomar la oferta educativa de la programación de Aprende en casa, en donde resalta la participación de profesores hablantes de español y, por tanto, se daba el uso exclusivo de esta lengua para la enseñanza. Y, en el mejor de los casos, la presencia de alguna lengua originaria, gracias a la participación de un profesor bilingüe, se daba bajo condiciones asimétricas. De tal modo, que el uso del español en la programación ocupo el lugar de la lengua de conocimiento, del discurso formal, y científico.

Por ejemplo, al observar el contenido abordado en la materia denominada "Lengua materna», notamos que se hace referencia a la existencia de otros idiomas que coexisten con el español. No obstante, no se trata de una clase de reflexión lingüística de una lengua originaria en particular, dirigido a los usuarios de este idioma. Más bien, el objetivo central parece ser la traducir 
de una lengua indígena al español, y viceversa. Por tanto, se descontextualiza su uso, y el reconocimiento de la cultura de sus hablantes. Y, en el caso de la LSM, además de no hablar sobre sus características como sistema lingüístico, tampoco se contó con un maestro sordo que apareciera en la pantalla, para que además de fortalecer la lengua materna se favoreciera la identidad sorda. Cabe destacar que esta situación no es exclusiva del programa Aprende en casa.

La gran ausente en el diseño del plan curricular de educación básica orientado a los alumnos sordos es la materia encaminada a la reflexión de la LSM como lengua materna, y, como era de suponerse, tampoco se pensó en incluirla en la temática de Aprende en casa. Sin embargo, habría que decir que la LSM sí se visibilizó, gracias a la presencia el intérprete de la lengua de señas (ILS) que aparecía en un recuadro en la pantalla del televisor. Aunque esta medida pudo beneficiar a la comunidad sorda en su acceso a la educación, resulta cuestionable para la enseñanza de los niños sordos de educación básica, porque como veremos más adelante, son nińos que, en su mayoría al provenir de hogares oyentes, no han adquirido una primera lengua, no han consolidado la gramática de la LSM, pero tampoco la del español, lo cual al iniciar su escolaridad los coloca en una situación de desventaja con respecto a sus pares oyentes.

En este trabajo discuto la compleja situación a la que se enfrentan los alumnos usuarios de una lengua minoritaria, con un énfasis en los sordos usuarios de la LSM, cuya enseñanza bilingüe bicultural/intercultural se vio amenazada ante la crisis educativa ocasionada por la pandemia. La respuesta del estado mexicano para continuar con la educación de este alumnado hizo patente la discriminación hacia los usuarios de una lengua diferente al español, al ser esta la lengua vehicular para la programación de Aprende en casa. Y, en el caso de los sordos, puso en evidencia el desconocimiento sobre la LSM y sus usuarios, en particular de la situación de los niños, quiénes ante la falta de modelos lingüísticos, sin un input lingüístico adecuado al interior de los hogares oyentes, aislados de sus pares sordos, no tuvieron un ambiente favorable para su desarrollo lingüístico, cognitivo y social.

En resumen, la gran pregunta es si en el diseño de la programación de Aprende en casa primó la mirada del español como la lengua dominante, y se idealizó al alumno urbano hablante del español como el público meta. Minimizando así el papel de la educación bilingüe intercultural, y con ello la búsqueda de una propuesta que beneficiara a los estudiantes de comunidades lingüísticas minoritarias, sordos y oyentes. En el desarrollo de esta exposición establezco un dialogo entre la educación bilingüe para las comunidades indígenas y para las comunidades sordas, y cómo la crisis sanitaria demostró la vulnerabilidad de su enseñanza. 


\section{Desarrollo}

\subsection{La educación bilingüe de los usuarios de lenguas minoritarias}

México es una nación plurilingüe y multicultural, es uno de los países de América Latina con el mayor número de lenguas originarias. A esta riqueza lingüística, compuesta de 364 lenguas indígenas además del español, habría que agregar el uso de dos lenguas de seńas que hasta el momento se han documentado, la lengua de señas mexicana (LSM), reconocida y empleada en todo el territorio nacional, y la lengua de señas maya yucateca (LSMy) cuyos usuarios se localizan en algunas comunidades de la península de Yucatán.

Ante tal diversidad lingüística el tema de la educación bilingüe bicultural/ intercultural resulta por demás complejo. Barriga (2018), al analizar la política lingüística de México en el periodo de 1992 a 2007, hace referencia a una serie de acontecimientos políticos y sociales que trastocaron el camino para la consolidación de las políticas lingüísticas de este país, desde la celebración del quinto centenario del descubrimiento de América (1992), hasta las reformas que se hicieron en la Constitución política de los Estados Unidos Mexicanos para declarar la pluriculturalidad y el multilingüismo de la nación mexicana en la primera década del siglo XXI. Para esta autora, estos hechos condujeron a cambios significativos que propiciaron la creación de valiosas instituciones, como la Coordinación General de Educación Intercultural Bilingüe y el Instituto Nacional de Lenguas Indígenas. Y, la propuesta de cambios en la legislación y normatividad en el ámbito educativo, que se cristaliza con el denominado Acuerdo 592 (Secretaría de Educación Pública-SEP, 2011).

Este encadenamiento de sucesos hace explícita la obligación del Estado para garantizar la educación de los pueblos indígenas a partir de un modelo bilingüe e intercultural. Sin embargo, como menciona Barriga (2018), la propuesta bilingüe intercultural que surge de este contexto no ha tenido los resultados esperados por varias razones, entre ellas: a) por la falta de una formación adecuada del profesorado para ejecutar el modelo bilingüe; b) por los múltiples prejuicios que persisten sobre las lenguas indígenas y sus usuarios, los cuales provienen tanto del exterior, si hablamos de una mirada occidental, como del interior, desde el discurso de los propios indígenas, quienes dada la violencia simbólica que de manera permanente se ejerce sobre este sector de la población, pueden otorgar un mayor valor a la enseńanza y uso del espańol, en menoscabo de su lengua materna y de su cultura; y c) por las condiciones estructurales de las escuelas primarias indígenas, carentes de recursos físicos (espacios adecuados, materiales didácticos) y humanos, que son necesarios para ofertar la educación que requieren los alumnos de las comunidades indígenas. 
Aunado a los factores enunciados, habría que destacar la incongruencia con respecto a la ubicación del docente que trabaja en el esquema de educación bilingüe en México. Destacamos el hecho que los profesores bilingües se caracterizan por su pertenencia a la comunidad en donde laboran, lo cual lograron a partir de los vínculos que establecen más allá de los padres de familia de los alumnos a los cuales enseña. Es decir, el trabajo del profesor rebasa el espacio escolar, y se convierte en un agente de cambio para el desarrollo de las comunidades en las que participa. Por tanto, llama la atención que por cuestiones administrativas los docentes bilingües (hablantes de una lengua indígena y del español), sean enviados a enseñar a lugares cuya la población tenga una lengua indígena diferente a la lengua materna del maestro.

Así, un maestro hablante de amuzgo puede ser enviado a comunidades en donde la lengua sea el mixe, o el chatino. Esto implica, en el mejor de los casos, que el docente aprenda el idioma de la comunidad (por un proceso de inmersión), pero, también, por el contrario, puede ocurrir que el maestro opte por usar el español como la lingua franca para enseñar e interactuar con su entorno. Al respecto, cabe mencionar que no es inusual que el uso exclusivo del español en la escuela se dé con la anuencia de los padres de familia, quienes prefieren que la enseńanza se haga en español. Este idioma se coloca como la lengua de prestigio a partir de la cual debe realizarse la construcción del conocimiento.

Me sumo al dicho de Barriga (2018) al considerar que todas estas razones, en su conjunto, nos conducen a pensar que el bilingüismo se reduce a una mera ilusión imposible de alcanzar. Lamentablemente, esta situación educativa la comparten las comunidades sordas en México. Desde hace dos décadas se puso en marcha el modelo educativo bilingüe bicultural (LSM-español). $\mathrm{Al}$ respecto podemos decir que al igual que en la atención a los alumnos indígenas, persiste la falta de profesores oyentes competentes en el uso de la LSM, y la carencia de profesores sordos competentes en español. Así como los prejuicios en torno a los sordos y a las lenguas de señas (LS); y al desconocimiento de los padres de familia (oyentes) sobre la importancia de la adquisición de una primera lengua, la LS. Esto en su conjunto condiciona que el objetivo de atención del niño sordo sea el aprendizaje del español oral, con los problemas que implica que esto se logre de manera eficaz.

Así que hoy vemos con sorpresa que la tendencia en la planeación educativa para la atención del alumnado sordo, no sea la formación de docentes sordos y oyentes, o la orientación a los padres de familia sobre la sordera y la LS, sino que la apuesta educativa recae en la presencia de un intérprete de lengua de señas (ILS) en el aula como la única forma de garantizar el acceso de 
la educación del alumnado sordo señante en el sistema de educación regular (Cruz-Aldrete, 2009; Cruz-Aldrete y Villa Rodríguez, 2016; Cruz-Aldrete, 2018; Obregón-Rodríguez y Valero, 2019).

\subsubsection{El desplazamiento de las lenguas originarias, ¿un problema educativo?}

En la actualidad presenciamos un movimiento generado por las propias comunidades para la conservación y transmisión de sus lenguas, aunado al apoyo de organismos internacionales, entre ellos, la Organización de las Naciones Unidas (ONU) cuya Asamblea General proclamó a 2019 como el Año Internacional de las Lenguas Indígenas. A través de la coordinación de la Organización de las Naciones Unidas para la Educación, la Ciencia y la Cultura (Unesco), se realizaron una serie de actividades a nivel mundial para sensibilizar a la opinión pública sobre los riesgos a los que se enfrentan estas lenguas originarias, que se han visto minorizadas como el vehículo para la transmisión de la cultura, negando, asimismo, su papel como rasgo de identidad de sus usuarios.

Aun cuando celebramos estas iniciativas, y los compromisos y acciones que los gobiernos a nivel mundial asumieron sobre este tema, en los hechos, no podemos obviar que las estrategias para la preservación de las lenguas vernáculas no han dado los resultados esperados. Si bien, es notorio que hay una mayor conciencia entre la población sobre las graves consecuencias que acarrea la pérdida de una lengua, no solo en términos de la diversidad lingüística, sino en las varias formas de conceptualizar el mundo, persiste el acelerado ritmo de la desaparición de las lenguas vernáculas.

En las primeras décadas del siglo XXI, hemos sido testigos de noticias que hacen referencia a la extinción de varias lenguas indígenas, a causa de la muerte de los últimos usuarios de estos idiomas. Esta información suele difundirse en distintos medios de comunicación, que, si bien revela la sensibilidad de la sociedad hacia la perdida de las lenguas nativas de México, también nos muestra el pulso de las políticas públicas para preservar estos idiomas. Se trata de noticias alarmantes, pero que no causan sorpresa alguna, puesto que, desde hace ańos, el Instituto Nacional de Lenguas Indígenas (INALI) (2012) ha manifestado el peligro en el que se encuentran varias lenguas de nuestra nación. Por mencionar un ejemplo, el libro titulado México. Lenguas indigenas nacionales en riesgo de desaparición publicado por el INALI (2012), discute sobre las precarias condiciones en las que se encuentran y, con ello, la gran posibilidad de su extinción. Sin duda, habría que seguir preguntándonos sobre las razones que han orillado a la desaparición de algunas de las lenguas utilizadas en este país. 
En principio, podríamos decir que la preservación y conservación de las lenguas depende del uso de estas en todos los espacios de la vida pública. Así, en un país como México, donde no hay una lengua oficial sino lenguas nacionales, tendría que ponderarse el valor de las sociedades multilingües. Y con ello, reconocer que hay personas usuarias de más de una lengua, y, por tanto, que es necesario discutir qué hacer para que el español no sea identificado como la única lengua de prestigio o la lengua de conocimiento.

Quizá la respuesta esté en la propia educación. Sí, en la puesta en marcha de un modelo bilingüe, pero con una perspectiva crítica, como lo plantean Daniel Heiman y Luis Urrieta (2019) en Educación bilingüe crítica. Estos autores destacan el elemento político de este tipo de educación. Son enfáticos al mencionar que los programas bilingües para grupos etnolingüísticos minorizados no promueven el bilingüismo a largo plazo, ni el biculturalismo, pues reconocen que la gran mayoría de los programas que analizaron suelen desvalorizar las lenguas e identidades originarias a favor de la lengua dominante hegemónica, y la identidad «normativizada» como la nacional. Si bien su análisis se centra en la educación bilingüe que se oferta en Estados Unidos para las poblaciones hispanohablantes, cuya mayoría está integrada por latinos de escasos recursos, es posible establecer un diálogo entre sus hallazgos, críticas, y reflexiones, con respecto a las características que se observan en la educación bilingüe que se imparte a las comunidades lingüísticas minoritarias en México, ya sean sordas o indígenas. Por ejemplo, mencionan que tanto docentes como alumnos comparten el prejuicio sobre el aprendizaje y fortalecimiento del idioma dominante, en detrimento de la otra lengua, bajo el supuesto que el dominio del inglés les brindará mejores oportunidades educativas y laborales. La situación descrita es similar a la que se vive en nuestro país con el aprendizaje y uso del español.

Cabe mencionar que, a diferencia de las comunidades indígenas, el reconocimiento de las personas sordas con miembros de una comunidad lingüística minoritaria es una conquista ocurrida en tiempos recientes. Así, el derecho de los sordos a ser enseñados en su propia lengua decantó en la propuesta de un modelo bilingüe bicultural (Peluso y Balieiro, 2015). Sin embargo, en el caso de México, este modelo pareciera que nació sin una clara política lingüística que diera cuenta de la diversidad de los miembros que conforman la comunidad sorda. Es decir, podemos encontrar al interior de este grupo, personas que han sido oralizadas con mayor o menor éxito, sordos que no han adquirido la LSM, o que no son fluentes en esta lengua, e incluso, individuos que solo utilizan señas caseras para su comunicación. 
La gran heterogeneidad lingüística de esta población demuestra que no se ha garantizado la adquisición de una primera lengua. $\mathrm{Al}$ respecto, no basta con reconocer que la sordera prelingüística dificulta el aprendizaje de la lengua oral, si no se ofrece de manera temprana un input lingüístico adecuado para los niños sordos. Y con ello, propiciar el desarrollo íntegro de estos individuos. Se ha demostrado la importancia de adquirir una primera lengua en los primeros cinco ańos de vida, pues a partir del uso de un sistema lingüístico se nombra y se construye el mundo. Se comparten pensamientos y emociones a partir de una lengua que se comparte con el otro. Pero, qué pasa con los niños sordos que provienen de hogares oyentes, de familias que ignoran la situación de la sordera, que no han tenido contacto con algún sordo, y, por tanto, desconocen la lengua y la cultura de la comunidad sorda. Sin duda, todo esto en su conjunto impacta de manera negativa en el desarrollo de la persona sorda, limitan la posibilidad del contacto con sus pares, y por consecuencia en la adquisición de su lengua natural, la LS.

La aún vigente discusión sobre la adquisición temprana de la lengua de señas versus la lengua oral dominante revela la situación por demás compleja, que viven los niños sordos, como lo han expuesto desde hace varias décadas diversos investigadores (Humphries et al., 2014), quienes atraen la atención de los especialistas y de la comunidad en general, sobre las delicadas condiciones en las cuales permanece la niñez sorda para adquirir una primera lengua. Situación que impacta además en su escolaridad.

$\mathrm{Al}$ ingresar a la escuela primaria, los niños oyentes cuentan con la gramática de su lengua materna, a diferencia de la gran mayoría de niños sordos que inician su escolaridad cuando aún no han dominado la gramática de una primera lengua. Por tanto, la complejidad de la educación bilingüe para la comunidad sorda resulta de entender que hay un problema lingüístico de base. Un alto porcentaje del alumnado sordo que llega a la escuela no es fluente en lengua de señas, pero tampoco es competente en el español, de ahí que la escuela se convierta en el primer espacio para el contacto entre pares, y sea el escenario que les brinda el input lingüístico para la adquisición de la LS.

Es la escuela donde a partir de la implementación de un modelo bilingüe bicultural se ha favorecido la adquisición de la LSM. Sin embargo, observamos que este modelo educativo no ha logrado que los alumnos aprendan el español (en su forma escrita) como una segunda lengua. De acuerdo con los datos reportados en Buendía (2014) por la Encuesta Nacional de la Dinámica Demográfica (ENADID), en el país existen 2,4 millones de mexicanos sordos, de los cuales, 84957 son menores de 14 años. De estos, solo 64\%, es decir 54372 , asiste a la escuela. Asimismo, se reconoce que uno de los sectores de 
la población más rezagada son los jóvenes sordos de entre 15 y 29 años, de los 124554 con esta discapacidad, $28 \%$, es decir 34875 , no tuvieron ningún tipo de educación. Y, desafortunadamente, para los 597566 sordos en edad adulta, que tienen entre 30 y 59 ańos, el contexto no es tan diferente. El 14\% nunca fue a la escuela y dos terceras partes (400 369) solo estudió hasta nivel básico (primaria y secundaria).

Es muy probable que la falta de un dominio de la lengua escrita sea una variable que condicione el acceso al nivel educativo medio superior y superior. Pero, también, como mencionábamos, al hecho que las personas sordas que cursan los primeros ańos de escolaridad no cuenten con una primera lengua. $\mathrm{Al}$ respecto, la crisis causada por la COVID-19 colocó en una situación de mayor vulnerabilidad a los estudiantes sordos usuarios de la LSM, en particular a los niños, debido a que los condujo a una situación de aislamiento.

Si tomamos en cuenta que la escuela es el punto de contacto con sus pares, debido a que la mayoría proviene de hogares oyentes en donde sus miembros no suelen utilizar la lengua de señas para comunicarse, los niños no tuvieron la oportunidad interactuar a través de esta. Y, si a esto le sumamos que entre las medidas que fueron implementadas para dar continuidad a la enseñanza de los alumnos que cursan la educación básica, a partir de la programación ofertada en Aprende en casa; Regreso a clases Aprende en casa II; Aprende en casa III, su experiencia educativa a partir del uso de la LSM no fue la adecuada, pues el discurso en esta lengua la más de las veces no correspondía a la competencia lingüística de los alumnos. Era indispensable el acompañamiento de los maestros oyentes usuarios de la LSM y de los maestros sordos, para abordar, complementar y enseñar los contenidos curriculares que se ofrecían en los diversos programas de Aprende en casa. Los padres oyentes suelen no tener dominio de la lengua de seńas, o su competencia es básica, no podría esperarse que tuvieran la misma participación en el proceso enseñanza-aprendizaje de sus hijos, como sí ocurrió con los padres oyentes con hijos oyentes, cuando ambos son usuarios del español.

Es decir, no hubo una planeación pensada para los estudiantes sordos señantes. No se consideró que un gran sector del alumnado al ingresar a la escuela está en el proceso de adquisición de la lengua de señas, que aún en grados superiores $\left(5^{\circ}\right.$ o $6^{\circ}$ ańo de escolaridad) no son fluentes en LSM, que presentan dificultades en la lengua escrita (español), y por tanto se necesita una didáctica orientada a las condiciones de esta comunidad. Consideramos que el acceso a la educación de las personas sordas bajo este esquema de Aprende en casa, con la sola aparición del intérprete de lengua de señas (ILS), no es el medio que garantiza colocar al alumno sordo señante en las mismas condiciones que su par oyente, como veremos más adelante. 


\subsection{Aprende en casa II (El discurso oficial)}

El Ciclo Escolar 2020-2021 inició el lunes 24 de agosto a través del programa de aprendizaje a distancia Aprende en casa II, para el cual la Secretaría de Educación Pública (SEP) (2020a) elaborará y transmitirá 2 mil 111 programas educativos, en una estrategia de enseńanza que llega a 30 millones de alumnos diariamente con el acompañamiento de televisoras privadas, de la red de radiodifusoras y televisoras educativas del país, así como de los sistemas públicos de comunicación del Estado mexicano. Los medios públicos y privados no tienen ninguna participación en el desarrollo de contenidos.

El secretario de Educación Pública Moctezuma Barragán precisó que los docentes elaboran los guiones de cada programa con base en los planes y programas de estudio vigentes (dotándolos de validez oficial) con la asesoría de conductores de la Dirección General de Televisión Educativa, para establecer el lenguaje de televisión necesario para este tipo de modalidad (Secretaría de Educación Pública-SEP, 2020b). Con base en datos presentados por el secretario de Educación Pública, la implementación de este programa aseguraría una educación con equidad ya que el $94 \%$ de las familias mexicanas tiene acceso a la televisión (Secretaría de Educación Pública-SEP, 2020a). Además, se establecerían tres semanas de reforzamiento a manera de curso de nivelación, para contrarrestar el rezago educativo que pudiese presentarse en el alumnado.

De acuerdo con datos de la Secretaría de Educación Pública-SEP (2020b) (Boletín 240) el programa Regreso a Clases. Aprende en casa II abarca a una población escolar total de más de treinta millones de educandos, de los cuales casi cinco millones corresponden a Preescolar; catorce millones a Primaria; casi 6,5 millones a Secundaria, y poco más de cinco millones de alumnos a Educación Media Superior. Este programa ${ }^{1}$ se trabajará a partir de cinco modalidades:

- Libros de texto gratuitos

- Programas de televisión

- Radio (transmitirá 640 programas de Educación Básica en 22 lenguas indígenas)

1 La implementación de este programa educativo a distancia también se complementa con apoyo socioemocional a alumnos y docentes con el fin de detectar posibles casos de deserción escolar; además de un contenido en la programación titulado Amar a México (Toribio, 2020), destinado a mostrar la riqueza cultural y natural de cada entidad de la República; contenido para «reforzar la educación indígena y especial", y la habilitación del Centro de Apoyo Pedagógico a Distancia, que brinda asesoría pedagógica y académica en Educación Básica por medio de 160 líneas telefónicas. 
- 350 mil cuadernillos impresos del Consejo Nacional de Fomento Educativo (CONAFE)

- Contenidos de internet que se difundieron en un principio en la página www.laescuelaencasa.mx y posteriormente en el portal del gobierno de México, en esta dirección: https://aprendeencasa.sep.gob.mx

En este nuevo escenario en el que la educación se apoya en la tecnología y medios de comunicación, el uso de la televisión cobra especial relevancia. $\mathrm{Al}$ respecto, Rebolledo (2011) nos muestra que los datos sobre el consumo de televisión en México revelan que el 98,3\% de los hogares urbanos mexicanos cuentan con un aparato de televisión, y que es considerado como el instrumento con fines educativos que más se utiliza debido al alcance que tiene este medio en la población mexicana.

Ahora bien, el uso de la televisión para

transmitir contenidos educativos, no es algo novedoso, si pensamos que en México la instancia denominada "Televisión educativa" data de la década de los 70' del siglo pasado. La Televisión educativa es un órgano centralizado que forma parte de la SEP, el cual tiene entre sus funciones el producir, programar y trasmitir contenidos educativos por medio de la televisión con el fin de desarrollar y fortalecer la enseñanza en nuestro país (Mendoza, 2013).

En su mayoría, el contenido de la televisión educativa se distribuye por sistemas de acceso restringido vía satélite o por cable, videoconferencias, videocasetes y aplicaciones multimedia, aunque algunas de sus producciones llegan también a sistemas de televisión abierta. La oferta de programación que se muestra es variada, incluye documentales, reportajes, entrevistas, informes, noticias, y programación infantil de corte lúdico.

A partir de la década de 1970, la televisión educativa de producción estatal adquiere mayor fuerza con programas y organismos públicos tales como la Unidad de Telesecundaria, la Unidad de Televisión Educativa y Cultural, el Instituto Nacional para la Educación de Adultos (INEA), Televisión de la República Mexicana, y Canal 22. Asimismo, uno de los principales proyectos generados por esta iniciativa la creación de la Red Satelital de Televisión Educativa (Red EDUSAT). Esta red de televisión de la SEP cuenta con una capacidad para transmitir hasta dieciséis canales de televisión, con programación cultural y educativa vía satélite.

Una de las metas de EDUSAT ha sido abatir el rezago educativo en México con la programación, transmisión y creación de programas educativos para televisión y radio acordes con las necesidades de diferentes públicos, así como con la formación, capacitación y actualización de profesionales vinculados con 
los medios audiovisuales educativos. Sin embargo, habría que mencionar que son escasos los contenidos que se encuentran realizados en lenguas originarias. $\mathrm{Y}$, que en pocas poblaciones rurales o indígenas se cuenta con la infraestructura para el uso de sus materiales.

El estado mexicano oportunamente utilizó la infraestructura que desde la década de los setenta del siglo pasado había construido a partir de la instancia de televisión educativa, para implementar la estrategia Aprende en casa, como aparece en el sitio https://www.televisioneducativa.gob.mx; sin embargo, insistimos que esto no es suficiente si no se toman en cuenta las condiciones de la población a la que va dirigida esta programación, como se ilustra con el caso de la comunidad sorda, en el siguiente apartado.

\subsubsection{El programa Aprende en casa y los niños sordos ¿Quién ha hablado por ellos?}

El 24 de abril de 2020 se vive una situación sin precedentes la SEP transmite dentro del programa Aprende en casa el contenido de un canal de televisión de Argentina dirigido a niños sordos señantes, la lengua vehicular de dicho contenido era la lengua de señas argentina (LSA). ¿Por qué razón se transmitió dicho programa en LSA y no en LSM?

De inmediato surgieron las voces de protesta de la Federación de Sordos de México, de líderes de la comunidad sorda, de maestros bilingües, de padres de familia, de los intérpretes de lengua de señas (ILS), por la utilización de materiales en LSA en la enseñanza de los niños sordos señantes de la LSM. La SEP cuestionada por esta situación, emite una respuesta manifestando que «incluyó dicho video por la identificación de actividades cotidianas, responsabilidades y organización del tiempo, de nińas y nińos, en casa y en la escuela, que se comparten con otras culturas». Y añaden,

Con este video se muestra a nińas y nińos mexicanos que existen otras formas de comunicación tanto verbal como a través de la lengua de señas siendo, en Argentina y en México muy similares y entendibles, al igual que el español que se habla en muchos países, el idioma tiene variaciones dialectales en su forma oral y señada (Diario de Yucatán, 24 de abril de 2020).

No queda más que sorprendernos por lo desafortunado de las declaraciones, y por revelar el profundo desconocimiento de las autoridades educativas de México, sobre el hecho que cada lengua de señas tiene una gramática particular, diferente a otra lengua de modalidad viso gestual. Sin dejar de lado, que también invisibilizó a la comunidad sorda de nuestro país. Esto ilustra la falta de claridad sobre la política lingüística y educativa para los sordos usuarios de una lengua de señas. 
Katia D'Artigues (2020), periodista mexicana y activista por el reconocimiento de los derechos humanos de las personas con discapacidad, en el sitio web Yo también, destaca la noticia «¡Ya hay lengua de señas en Aprende en casa! (y la SEP hará más)». Escribe lo siguiente en el primer párrafo: «Este lunes 4 de mayo, al fin, en los programas destinados a preescolar y primaria del programa Aprende en casa ya contienen una persona intérprete en lengua de señas mexicana. La próxima semana también lo tendrán los contenidos de secundaria a partir del 11 de mayo». Y posteriormente, en el segundo párrafo, agrega que Marcos Bucio, Subsecretario de educación básica, ha declarado que: «la SEP atiende a niñas y niños con discapacidad de educación básica, a través de una estrategia de educación inclusiva que se incorpora de manera gradual al programa Aprende en casa».

\section{Conclusiones}

La implementación de la propuesta Aprende en casa para dar continuidad a la educación de los niños y jóvenes de México, ha demostrado, lamentablemente, la inequidad de la educación que se oferta en nuestro país, la gran desigualdad social, y la discriminación. El precario equilibrio en que se encuentra la educación bilingüe instrumentado para las comunidades con una lengua originaria, y las comunidades sordas, las coloca en una peligrosa intersección entre el fortalecimiento de la lengua materna y el aprendizaje de la lengua dominante. Y de manera particular, la atención al alumnado sordo cuya atención no logra a definirse en términos de interculturalidad o de educación especial.

Si bien, el programa Aprende en casa es una iniciativa y esfuerzo plausible emanado de la SEP, que surgió de la emergencia sanitaria, no podemos negar que hubo una omisión hacia las comunidades usuarias de una lengua minoritaria. Celebramos que la SEP haya atendido a la demanda de contar con interpretación de LSM en la barra de programas de Aprende en casa II. Sin duda la labor de los ILS es fundamental para la atención de las personas sordas. No obstante, es necesario cuestionarnos por qué la tarea educativa, el acceso a la educación descansa en la participación de los ILS, y no en la participación de todos los involucrados, docentes (sordos y oyentes), familia, autoridades educativas. Aun no se entiende que el gran problema educativo del alumnado sordo parte del derecho a utilizar y fortalecer su propia lengua, la LSM. Y, por tanto, que el objetivo principal en este periodo de confinamiento en casa habría sido proporcionar un espacio por televisión para que la familia oyente conociera y aprendiera esta lengua, en compañía de sus hijos sordos. 
Por otra parte, no podemos continuar con la inercia de pensar que la educación a distancia para el alumnado se resuelve por el solo uso de plataformas digitales, o a través de la implementación de programas educativos por televisión abierta (Secretaría de Educación Pública-SEP, 2020c) (boletín 267). La crisis causada por la pandemia provocó una mayor crisis educativa, un mayor índice de deserción. Y, que, en el caso de las comunidades indígenas y sordas, trajo como consecuencia que el espacio ganado para la instrumentación de una enseñanza bilingüe bicultural se viera difuminado.

Observo que se corre el riesgo de afianzar la idea que la presencia del ILS garantiza el acceso a la educación del sordo (ya sea en el aula presencial o en los medios digitales), puesto que hay quienes piensan que contar con un espacio para la interpretación de la LSM, como en el programa Aprende en casa, es suficiente para atender la demanda de enseñanza para las personas sordas señantes. Negando así que el primer objetivo de la educación de los niños sordos es la adquisición de una primera lengua, y este proceso no se da a través de la interpretación/traducción de un contenido curricular que explica en español y se traduce a la LSM.

Redefinamos los objetivos de la educación en el contexto que hoy nos toca vivir. Hay una oportunidad de aprovechar este episodio en la educación de las comunidades lingüísticas minoritarias, que nos debe conducir a explorar nuevas formas de enseñanza, a aprender de otras maneras, a repensar el modelo educativo que se ofrece a los hablantes de lenguas originarias, a los sordos señantes. No debemos dejarla ir. Lograr un mundo en donde quepan muchos mundos, inicia con la educación de todos sus habitantes sean usuarios de una lengua oral o de una lengua de señas.

\section{REFERENCIAS BIBLIOGRÁFICAS}

Barriga, R. (2018). De Babel a Pentecostés. Politicas lingüisticas y lenguas indigenas, entre historias, discursos, paradojas y testimonios. México: Secretaría de Educación Pública, Coordinación General de Educación Intercultural y Bilingüe.

Buendía, E. (02 de abril de 2014). Sordos: sin educación ni trabajo. El universal. Recuperado de https://www.eluniversal.com.mx/articulo/periodismo-dedatos/2017/04/2/sordos-en-mexico-sin-educacion-ni-trabajo

Cruz-Aldrete, M. (2009). Reflexiones sobre la Educación bilingüe Intercultural para el sordo en México. Revista Latinoamericana de Educación Inclusiva, 3(1), 133-145.

Cruz Aldrete, M. (2018). Manos que anidan paciencia. Revista de Administración Pública, 52(1), 177-189. 
Cruz-Aldrete, M., y Villa Rodríguez, M. A. (2016). Middle School Deaf Education in Mexico: A Postponed Issue. En B. Gerner de García y L. Becker Karnoop (eds.), Change and promise. Bilingual Deaf Education and Deaf Culture in Latin America (pp. 106-121). Washington DC: Gallaudet University Press.

DiariodeYucatán(24abrilde2020).SEPtransmiteprogramaparaniñossordosenlengua de señas argentina. Recuperado de https://www.yucatan.com.mx/mexico/ sep-transmite-programa-para-ninos-sordos-en-lengua-de-senas-argentina

D’Artigues, K. (5 de octubre de 2020). ;Ya hay lengua de señas en Aprende en Casa! (y la SEP hará más). Yo también. Recuperado de https://www.yotambien. $\mathrm{mx} /$ notas/ya-hay-lengua-de-senas-en-aprende-en-casa/

Heiman, D. y Urrieta L. (2019). Educación bilingüe crítica. En I. Villegas, Gunther Dietz, M. F. Saavedra (coords.), La traducción lingüistica y cultural en los procesos educativos: hacia un vocabulario interdisciplinary (pp. 111-133). México: Universidad Veracruzana, Universidad Nacional Autónoma de México.

Humphries, T., Kushalnagar, P., Mathur, G., Jo Napoli, D., Padden C. y Rathmann, C. (2014). Ensuring language acquisition for deaf children: What linguists can do. Language, 90(2), e31-e52. https://doi.org/10.1353/ lan.2014.0036

Instituto Nacional de Lenguas Indígenas. (2012). México. Lenguas indígenas nacionales en riesgo de desaparición. Arnulfo Embriz Osorio y Óscar Zamora Alarcón (coords.). México: INALI.

Mendoza, I. (2 de septiembre de 2013). Televisión Educativa. Universidad Tecnológica Latinoamericana en Linea (UTEL). Recuperado de https:// www.utel.edu.mx/blog/10-consejos-para/television-educativa/

Obregón-Rodríguez, M., y Valero Weeke, M. (2019). Best Practices and Challenges of Deaf Educaton in Mexico. En H. Knoors, M. Brons y M. Marshark (eds.), Deaf Education Beyond the Western World. Context, Challenges, and Prospects. Nueva York: Oxford University Press. https://doi.org/10.1093/ oso/9780190880514.003.0016

Peluso, L. y Balieiro Lodi, A. C. (2015). La experiencia visual de los sordos. Consideraciones políticas, lingüísticas y epistemológicas. Pro-Posiçōes, 26(3), 59-81. https://doi.org/10.1590/0103-7307201507803

Quishpe Lema, C. (2001). Educación intercultural y bilingüe. En Instituto Científico de Culturas Indigenas RIMAI, 3(31). Recuperado de http://icci.nativeweb. org/boletin/31/quishpe.html

Rebolledo, R. A. (11 de agosto de 2011). 9 Datos sobre el consumo de TV en México. El Economista. Recuperado de https://www.eleconomista.com.mx/empresas/ 9-datos-sobre-el-consumo-de-TV-en-Mexico-20160811-0092.html 
Secretaría de Educación Pública-SEP. (2011). Acuerdo 592 por el que se establece la articulación de la Educación. México: SEP-Dirección de Enlace y Vinculación-Dirección General de Desarrollo Curricular, Subsecretaría de Educación Básica. Recuperado de https:/www.sep.gob.mx/work/models/ sep1/Resource/9721849d-666e-48b7-8433-0eec1247f1ab/a592.pdf

Secretaría de Educación Pública-SEP (03 de agosto de 2020a). Boletín No. 205. Iniciará el Ciclo Escolar 2020-2021 con el modelo de aprendizaje a distancia Aprende en Casa II: Esteban Moctezuma. Recuperado de https:// www.gob.mx/sep/es/articulos/boletin-no-205-iniciara-el-ciclo-escolar2020-21-con-el-modelo-de-aprendizaje-a-distancia-aprende-en-casa-iiesteban-moctezuma?idiom=es

Secretaría de Educación Pública-SEP (29 de agosto de 2020b) Boletín No. 240 Produce SEP 4 mil 550 programas de televisión de Regreso a Clases. Aprende en Casa II: Esteban Moctezuma Barragán. Recuperado de https:// www.gob.mx/sep/articulos/boletin-no-240-produce-sep-4-mil-550-programas-de-television-de-regreso-a-clases-aprende-en-casa-ii-esteban-moctezuma-barragan?idiom=es

Secretaría de Educación Pública-SEP (07 de octubre de 2020c). Boletín No. 267 Confirma continuidad del programa Aprende en Casa II hasta que todo el país se encuentre en semáforo epidemiológico verde. Recuperado de https://www.gob.mx/sep/es/articulos/boletin-no-267-confirma-continuidad-del-programa-aprende-en-casa-ii-hasta-que-todo-el-pais-seencuentre-en-semaforo-epidemiologico-verde?idiom=es

Toribio, L. (18 de agosto de 2020). Asegura Esteban Moctezuma que programa "Aprende en casa II" contempla educación especial. Imagen Radio. Recuperado de https://www.imagenradio.com.mx/asegura-esteban-moctezuma-que-programa-aprende-en-casa-ii-contempla-educacion-especial

Villa-Rodríguez, M. A. (2017). Cognición y aprendizaje en las personas sordas. En M. Cruz Aldrete (coord.), Habla del silencio: estudios interdisciplinarios sobre Lengua de Señas Mexicana y la comunidad Sorda (pp. 199-209). México: Bonilla Artigas/UAEM. 